\title{
Cultura de paz y expresiones artísticas con personas privadas de libertad en el Centro de Atención Institucional Calle Real (Liberia) y en el Centro Semi- Institucional (Nicoya)
}

\section{Culture of Peace and Artistic Expressions With Persons Deprived of Liberty in the Institutional Attention Center Calle Real (Liberia) and the Semi-institutional Center (Nicoya)}

Doriam Chavarría López

Universidad Nacional

Sede Regional Chorotega

Guanacaste, Costa Rica doriam.chavarria.lopez@una.cr

Juan Diego Gómez Navarro

Universidad Nacional

Heredia, Costa Rica

juan.gomez.navarro@una.cr

Helen Marenco Rojas

Universidad Nacional

Heredia, Costa Rica

helen.marenco.rojas@una.cr

Jenniee Vargas Cárdenas

Universidad Nacional

Sede Regional Chorotega

Guanacaste, Costa Rica jenniee.vargas.cardenas@hotmail.com 
ReVista Universidad en DiÁlogo • Vol. 9, N. ำ 1 , Enero-Junio, 2019 • 95-109

ISSN 2215-2849 • EISSN: 2215-4752

URL: http://www.revistas.una.ac.cr/index.php/dialogo/index CORREO ELECTRÓNICO: universidadendialogo@una.cr DOI: https://doi.org/10.15359/udre.9-1.6

Recibido: 27/04/2018 Aceptado: 12/09/18

Resumen. El proyecto que se expone a continuación es un complemento del proceso para la construcción de una cultura de paz dentro de los centros penitenciarios, cuyas acciones a realizar, de índole pedagógica, formativa y práctica, se orientan al fortalecimiento del conocimiento propio y del comportamiento social, a través de actividades fundamentadas en técnicas artísticas con base en el lenguaje del movimiento. Dado que lo que se busca es que las personas privadas de libertad tengan mayor conciencia de la importancia de su rol como personas y como individuos dentro de la sociedad, posibilitando una mejor resolución de los problemas a los que se enfrentarán una vez que se reintegren a la sociedad. Por medio de talleres, charlas y presentaciones artísticas, y a través de la reflexión y la conciencia de las situaciones cotidianas, se pretende que como seres humanos los participantes formen parte de procesos de socialización y de las construcciones sociales en las que se debe cumplir con modelos ideales de ser. Mediante la integración, la participación, la comunicación y la expresión corporal las personas privadas de libertad mejorarían la percepción que tienen de sí mismos, y lograrían generar formas alternativas de reflexionar sobre los problemas y su resolución; todo esto, a través del proceso de empoderamiento que se promueve en los participantes desde la ludopedagogía, que fortalece el proceso hacia una cultura de paz.

Palabras clave: cultura de paz, expresiones artísticas, acción participativa, convivencia, reinserción.

Abstract. The project presented below is a complement to the process of building a culture of peace within prisons, whose actions to be carried out in a pedagogical, formative, and practical way are aimed at strengthening one's own knowledge and social behavior, through activities based on artistic techniques based on the language of movement. What is sought is that people deprived of freedom have a greater awareness of the importance of their role as individuals and as individuals within society, by enabling a better resolution of the problems they will face once they reintegrate into society. Through workshops, talks and artistic presentations, and through reflection and awareness of everyday situations, it is intended that, as human beings, the participants form part of socialization processes and social constructions in which they must comply with ideal models of being. Through integration, participation, communication, and corporal expression, people deprived of liberty would improve their perception of themselves and generate alternative ways of reflecting on problems and their resolution; all this, through the process of empowerment that is promoted in the participants from the ludopedagogy, which strengthens the process towards a culture of peace.

Keywords: culture of peace, artistic expressions, participatory action, coexistence, reintegration. 
URL: http://www.revistas.una.ac.cr/index.php/dialogo/index

CORREO ELECTRÓNICO: universidadendialogo@una.cr

DOI: https://doi.org/10.15359/udre.9-1.6

\section{Introducción}

A partir de los esfuerzos colectivos que se han evidenciado desde el trabajo en el Sistema Penal Costarricense, por parte de la Universidad Nacional de Costa Rica (UNA), consideramos necesario caracterizar el panorama en el que se desarrolla el fenómeno penitenciario en el país.

En concordancia con la Declaratoria de las Universidades Estatales para el año 2017, que celebra la vida, el diálogo y la paz, los esfuerzos institucionales de la Educación Superior del Estado se orientan a generar propuestas de abordaje a las problemáticas sociales, mediante la creación de espacios para el fomento de la convivencia pacífica y el desarrollo de acciones que incentiven la búsqueda del bienestar social en todos los estratos que conforman la sociedad costarricense.

Nuestra percepción del nuevo humanismo, como corriente ético-filosófica cimentada en el quehacer académico y práctico del Centro de Estudios Generales de la Universidad Nacional, rechaza toda forma de exclusión, segregación y desigualdad social legitimada y reproducida por los agentes socializadores y traducidos desde la interacción en la cotidianidad en aras del desarrollo integral del ser humano y el entorno. Por lo que, a partir de esto, el estudio y abordaje del fenómeno de la pobreza en los países latinoamericanos es un tema cardinal en la tarea de analizar las causas de la conducta delictiva, las características del proceso penitenciario y del imaginario construido en torno a las personas privadas de libertad. Como lo señalan Dell' Anno y Teubal (2006):

(...) las marcas de la pobreza quedan impresas en el desarrollo psicofísico y las enfermedades carenciales, la falta de credenciales educativas, el vocabulario, la vestimenta, la falta de avales para ocupar determinadas posiciones, etc. Si a éstas se suma algún antecedente policial o penal, las oportunidades de superar la marginación se minimizan y el individuo entra en una situación de exclusión que puede ser definitiva. (p.158)

En Costa Rica, de acuerdo con los resultados del II Informe del Estado de la Justicia (2017), se constata que la mayoría de las personas condenadas con prisión efectiva proviene de grupos de población que viven en pobreza. En términos generales, los privados de libertad son hombres jóvenes pertenecientes a los estratos socioeconómicos más bajos. Hasta abril de 2016 las personas encarceladas eran sobre todo hombres (93\%), jóvenes (52\% menores de 35 años y $77 \%$ menores de 45 años) (p. 365). 
Revista Universidad en Diálogo • Vol. 9, N. ำ 1 , Enero-Junio, 2019 • 95-109

ISSN 2215-2849 • EISSN: 2215-4752

URL: http://www.revistas.una.ac.cr/index.php/dialogo/index CORREO ELECTRÓNICO: universidadendialogo@una.cr

DOI: https://doi.org/10.15359/udre.9-1.6

El Centro de Estudios Generales ha generado varias intervenciones por medio de proyectos en el Sistema Penitenciario Nacional, desde el año 2015 se incursiona por primera vez logrando un acercamiento a la experiencia de las personas privadas de libertad y explorando un abanico de múltiples manifestaciones conductuales, sociorelacionales y afectivas generadas en el grupo participante, orientadas a ser potencialidades y destrezas para la construcción de espacios de convivencia pacífica, de reflexión y aprendizaje, basado en el análisis del fenómeno penitenciario desde la vivencia de la reclusión en el Centro de Atención Institucional San Rafael.

Como resultado de estas primeras intervenciones, visualizamos en la población participante la tendencia hacia la autorrealización, la desmitificación de estereotipos socialmente sostenidos en torno a las personas privadas de libertad, la crítica constante al proceso penitenciario como un agente deshumanizante, el reconocimiento de la violencia como un mecanismo de sobrevivencia que se traduce en redes de apoyo que reproducen la opresión de grupos diferenciados y demás cuestiones que pasan de ser concebidas como hechos ineludibles a ser materia de problematización, es decir, que el espacio creado por las personas privadas de libertad para la relectura de la realidad penitenciaria deviene en la generación de insumos indispensables para promover una cultura de paz.

En lo que se denomina un segundo proceso, este año 2017 se inicia en forma conjunta entre el Centro de Estudios Generales y la Sede Regional Chorotega de la Universidad Nacional el proyecto Cultura de paz y expresiones artísticas con personas privadas de libertad en el Centro de Atención Institucional Calle Real en Liberia y en el Centro Semi-Institucional de Nicoya, enfocado en la creación de espacios para la socialización que permitan la visualización en torno al contexto en el que viven el proceso penitenciario las personas privadas de libertad y orientar los productos generados en este espacio para construir experiencias formativas para inculcar valores, actitudes, fortalecer la autoestima y desarrollar habilidades, por medio de talleres y charlas y utilizando el arte como estrategia para canalizar la energía y contribuir a liberar emociones, incentivando la creatividad.

Como una de nuestras pretensiones en este proyecto, queremos identificar, analizar y abordar los elementos determinantes en la evolución de las condiciones de los Centros de Atención Institucional para el proceso punitivo de las personas privadas de libertad: como lo son el hacinamiento y las malas condiciones de habitabilidad, temas urgentes que requieren solución por ser factores que atentan directamente contra la posibilidad de un verdadero proceso de resocialización y empoderamiento de las personas que conforman el sistema penitenciario. 
URL: http://www.revistas.una.ac.cr/index.php/dialogo/index

CORREO ELECTRÓNICO: universidadendialogo@una.cr

DOI: https://doi.org/10.15359/udre.9-1.6

Según el Consejo Nacional de Rectores (CONARE), en el II Informe Estado de la Justicia (2017), en referencia a la cantidad de privados de libertad en el Programa Institucional:

Para el mes de octubre del año 2017 se registra en el Programa Institucional, según datos del Ministerio de Justicia y Paz, un total de 842 personas en el Centro Institucional de San José, 1271 en el Antonio Bastida en la UAI Pabrú Presberi un total de 86 privados de libertad, en el Centro Gerardo Rodríguez se registran 1286 personas, en el programa de Adulto Mayor una cantidad 167 privados de libertad, la Reforma registra un total de 3004 personas, en el Centro Luis Paulino Mora un total de 1084 personas privadas de libertad, en la Unidad Integral de Atención Reinaldo Villalobos 305 personas, el CAI Nelson Mandela en San Carlos registra un total de 800 personas, en el centro Jorge de Bravo un total de 517 privados de libertad, Heredia registra 01 persona, Liberia aumenta su cifra con 1104 personas privadas de libertad, el Centro Institucional 26 de Julio 913 personas, en el Marcus Garvey 714 personas privadas de libertad, en el Carlos Luis Fallas 1209 personas. En conclusión, en el mes de octubre del año en curso se registra un total de 13303 personas privadas de libertad en el Programa Institucional. Datos directamente relacionados con el hacinamiento en centros penales que alcanzaba un $45.98 \%$ de sobrepoblación.

Agrega el II Informe Estado de la Justicia (2017),

Los privados de libertad en el Programa Semi Institucional, que el registro total según estadísticas del Ministerio de Justicia y Paz en el mes de enero del 2017, corresponde a 4708 personas, donde 1602 personas se encuentran en el Semi Institucional de San José, 284 en Pérez Zeledón, 33 en La Paz, en San Ramón un total de 314 personas, en Cartago 386 personas, 629 en Heredia, 122 en San Luis, Nicoya con 203 personas, Liberia con 328 personas, Puntarenas 345 personas y Limón 462 personas. Bajo el mismo programa de atención para el mes de octubre del presente año se registra un total de 4303 personas en centros Semi Institucionales, San José registra un total de 1403, Pérez Zeledón 271, La Paz 29, San Ramón, 269, Cartago 373, Heredia 626, San Luis 107, Nicoya 196, Liberia 257, Puntarenas 318 y Limón aumentó con 454 personas.

Los objetivos de nuestro proyecto responden a la necesidad de guiar el trabajo intelectual hacia posibles propuestas de solución a la problemática penitenciaria, elaboradas con rigor académico, criticidad, ética y responsabilidad social. 
Donde podamos generar los espacios que den inicio a la construcción de conocimiento liberador, donde se venzan estereotipos y se visibilice a la población penal de Costa Rica como agente de cambio y no como portadora pasiva del problema.

Queremos, de este modo, asumir el compromiso de trascender las formas tradicionales de abordar el fenómeno de la delincuencia, mediante la propiciación de un diálogo entre el sector académico y las personas privadas de libertad, un diálogo necesario para un acercamiento en la construcción de soluciones reales. Consideramos a la academia como responsable de garantizar que la producción de conocimiento esté al servicio de la sociedad para efectos de su liberación y que reconozca a las personas privadas de libertad como expertas desde la vivencia del fenómeno penitenciario, quienes a partir de su cotidianidad pueden convertirse en agentes primordiales para el cambio de este fenómeno.

\section{Cultura de paz desde una visión del conflicto}

El término cultura de paz es definido desde la concepción de distintos componentes, como el social, el jurídico y el cultural, mismos que se construyen dentro de la sociedad. De la misma manera, los seres humanos forman parte del sistema social que impone a la fuerza una serie de normas sociales que reprimen e inducen al ser humano a la violencia, ordenando y direccionando las vidas de las personas.

Según el análisis de Geertz (1989) desde la antropología, se entiende como cultura a una serie de directrices simbólicas que controlan la conducta, estas son transmitidas por medio de agentes socializadores durante la infancia, y son reafirmadas o transgredidas a lo largo de la vida de una persona. Tal como lo señala Vargas (2016):

(...) que en el caso de la violencia, es una conducta que tiende a estar reforzada por un sistema económico capitalista en la que fomenta la discriminación y la exclusión, sin embargo, las conductas sociales que se aprenden, también se pueden cambiar es por ello que se puede implementar espacios de convivencia pacífica, la violencia es una construcción social y de allí, deduce la paz, debería también ser una construcción social. (pp. 27-29)

Con base en esto, se plantea promover una cultura de paz desde una visión del conflicto, orientado bajo una percepción social, cultural y jurídica, ya 
URL: http://www.revistas.una.ac.cr/index.php/dialogo/index

CORREO ELECTRÓNICO: universidadendialogo@una.cr

DOI: https://doi.org/10.15359/udre.9-1.6

que donde se manifiesta conflicto es posible que se genere violencia. Según la definición de las Naciones Unidas (1998, Resolución A/52/13), la cultura de paz consiste en una serie de valores, actitudes y comportamientos que rechazan la violencia y previenen los conflictos tratando de atacar sus causas para solucionar los problemas mediante el diálogo y la negociación entre las personas, los grupos y las naciones.

Con los resultados logrados a partir de nuestras experiencias, nos oponemos a las concepciones tradicionales sobre la paz, que la reconocen como la ausencia de violencia, conflicto y confrontación, como si estos no fueran inherentes a la condición humana, y aún más importante, como si estos elementos no fueran base de la exclusión que se reproduce en la vida social en detrimento de grupos humanos postergados tradicionalmente en su pleno desarrollo.

Nuestra propuesta desde el proyecto Cultura de paz y expresión artística con personas privadas de libertad consiste en la búsqueda de un replanteamiento conceptual en el que se reconoce a la paz como el rechazo a toda forma de violencia, exclusión y marginación socialmente legitimada, así la definición de la cultura de paz se inclina hacia la resolución de los conflictos por medio de mecanismos alternos traducidos teórica y metodológicamente en cinco áreas:

a) El reconocimiento de aspectos determinantes de la historia de vida de las personas involucradas en el fenómeno

b) Mecanismos de negociación como herramientas para el logro de consensos en situaciones problemáticas

c) El estudio de la comunicación humana para la comprensión del contenido real de los discursos, argumentos y mensajes que se gestan en la dinámica social.

d) La resolución alternativa de conflictos como marco de acción que provee de herramientas para afrontar la confrontación y el conflicto, cuestiones inherentes a la condición humana, sin apelar a la violencia.

e) Promoción de una cultura de paz como una construcción colectiva que permite la convivencia pacífica. Un conjunto de operaciones simbólicas como costumbres, tradiciones, tendencias y conductas orientadas a buscar el bienestar común, hacia el crecimiento individual como interdependiente e interdefinido por las condiciones del entorno social. 
ReVista Universidad en DiÁlogo • Vol. 9, N. ำ 1 , Enero-Junio, 2019 • 95-109

ISSN 2215-2849 • EISSN: 2215-4752

URL: http://www.revistas.una.ac.cr/index.php/dialogo/index CORREO ELECTRÓNICO: universidadendialogo@una.cr

DOI: https://doi.org/10.15359/udre.9-1.6

Por lo anterior, creemos que dentro del marco del sistema penitenciario costarricense se deben plantear una serie de retos que se pueden asumir para construir un modelo de mayor inclusión, en donde se generen rupturas con un sistema social basado en la individualización, transgrediéndolo dentro de la institución penal, creando otros recursos para la convivencia en el proceso de reclusión basados en la identificación y el sentido de pertenencia. Consideramos que deben ser orientados a lograr lo siguiente:

- Reformular y reconceptualizar las nociones de readaptación, reinserción y libertad que rigen el quehacer del Ministerio de Justicia y Paz y, por ende, del sistema penitenciario costarricense.

- Incluir en el programa de atención a la persona privada de libertad en los centros penales, además de los componentes de formación académica y laboral, un apartado dedicado a fomentar el empoderamiento de las personas a partir del análisis de los condicionantes sociales que potencian la conducta delictiva, el reconocimiento de las fortalezas de la población penal para asumir un proceso de cambio colectivo y la organización de las personas para el progreso y desarrollo social.

- Invertir esfuerzos y recursos para la generación de redes de apoyo institucionalizadas a lo externo del centro penal para la prevención de la conducta delictiva y para el seguimiento de las personas exprivadas de libertad en pro de reducir los índices de reincidencia en el delito.

De igual forma, debemos prestar atención a la exclusión social que es, a nuestro juicio, la causa que hace posible la pobreza, la vulnerabilidad y la situación de riesgo. Es claro que la exclusión social es más que la escasez de dinero, supone el acceso limitado al capital humano (educación, trabajo, salud, participación social) y genera en las personas excluidas la construcción de mecanismos de sobrevivencia que violentan la ley, reproducen la violencia y producen un malestar generalizado en la sociedad. Desde nuestra experiencia entendemos que la exclusión se da a partir de la falta de oportunidades, la inequidad y la desigualdad legitimada. Por tanto, las condiciones sociales son determinadas por el contexto social, económico y político.

En Latinoamérica, las características socioeconómicas de la mayoría de personas que conforman la población privada de libertad evidencian que es gente que crece en comunidades tradicionalmente postergadas y marginadas. 
URL: http://www.revistas.una.ac.cr/index.php/dialogo/index

CORREO ELECTRÓNICO: universidadendialogo@una.cr

DOI: https://doi.org/10.15359/udre.9-1.6

Por ello, debemos prestar atención a los procesos post-penitenciarios, ya que estos son parte de los que concurren en el sistema penitenciario, el cual debe dar seguimiento a la persona privada de libertad que ha cumplido la pena y se enfrenta a las condiciones excluyentes del entorno social.

Estos procesos deben propiciar la creación de redes de apoyo comunitarias e institucionales. Con la ejecución del I Congreso Internacional "Cultura de Paz: por un Diálogo entre Personas Privadas de Libertad y el Sistema Penitenciario Costarricense", que se realizó el 22 y 23 de junio del 2017, se abrieron los espacios para orientar la discusión y determinar cuáles son las necesidades más urgentes de las personas al finalizar su proceso penitenciario y qué dificulta su pleno desenvolvimiento al reintegrarse a la vida fuera del penal, y que en muchas ocasiones contribuye a la reincidencia delictiva.

Respondiendo al objetivo estratégico de coadyuvar al proceso de inserción social para las personas que cumplen su sentencia privativa de libertad en un centro de atención institucional, nos hemos dado a la tarea de apoyar a la unidad organizacional orientada a la implementación de acciones que favorezcan el proceso de inserción social de un sector de la población penitenciaria sentenciada. Dadas las necesidades que presenta determinada población penitenciaria y de conformidad con las competencias atribuidas al Ministerio de Justicia y Paz, consideramos relevante la creación por parte del Ministerio de una estructura organizacional denominada Unidad de Inserción Social (UIS), que apoye los procesos de inserción social de las personas privadas de libertad una vez que cumplan su sentencia en un Centro de Atención Institucional, y que contribuya a la disminución de la reincidencia delictiva y a una mejoría en la percepción de la seguridad por parte de la ciudadanía.

La Unidad de Inserción Social es un medio de convergencia de diversos esfuerzos y recursos nacionales e internacionales tanto del sector gubernamental como privado, así como el aporte de la academia y organizaciones no gubernamentales, las cuales puedan contribuir a la gestión institucional, en particular al quehacer del sistema penitenciario y en materia de prevención, mediante el fortalecimiento de procesos coordinados y de ejecución de acciones. Desde nuestra perspectiva queremos resaltar dos funciones principales de esta iniciativa de la UIS, las cuales son:

a) El manejo de las relaciones públicas, con el fin de lograr la suscripción y ejecución de acuerdos, convenios, alianzas y obtención de cooperación nacional e internacional, centralizando y optimizando la información y oportunidades con las que se cuentan. 
b) Creación de planes, programas y proyectos de apoyo para la población meta, mediante diversas modalidades como la atención individual en los centros penitenciarios, la atención grupal e inclusive familiar, referencias necesarias de las personas beneficiadas hacia otras entidades, sean estas de la propia institución o de otras instituciones no gubernamentales.

Todo esto lo estamos proponiendo a partir del análisis del fenómeno desde la academia; sin embargo, creemos que es esencial el criterio del mismo Ministerio de Justicia y Paz y de los representantes del sistema penitenciario para la elaboración de retos que solo se pueden plantear desde la experiencia. A partir del proyecto hemos creado instrumentos que nos permiten estos acercamientos y una de estas experiencias fue la que construimos en forma conjunta en el congreso que realizamos en Liberia en el mes de junio, en la sede Chorotega de la Universidad Nacional. Visualizamos este congreso como una invitación para el planteamiento de retos nuevos generados entre el quehacer universitario, el trabajo en las instituciones y la vivencia de las personas privadas de libertad, lo cual nos guio para orientar el proceso de cambio que proponemos.

Con base en esto, estamos planteando construir una cultura de paz desde una visión del conflicto, orientado bajo una percepción social, cultural y jurídica, ya que donde se manifiesta conflicto se genera violencia. Como lo menciona Geertz (1989), en su artículo "El impacto del concepto de cultura en el concepto de hombre", dentro de la complejidad de la cultura de la sociedad, la paz puede ser descrita e interpretada desde muchas aristas, en este caso, es vista como la alternativa, y no la ausencia del conflicto; afirmando de la misma manera las múltiples consecuencias de un sistema represor que obliga al ser humano a adaptarse a las condiciones que este mismo genera frente a la misma complejidad.

Como se ha indicado antes, se considera necesario visualizar la condición excluyente de la que provienen las personas privadas de libertad y en la cual continúan desenvolviéndose una vez que han sido condenadas. Esto con el propósito de evidenciar, en la práctica, los efectos que resultan del sistema represor y que persisten dentro de los centros penitenciarios sobre las personas privadas de libertad.

Por ello, el aspecto jurídico en este proyecto plantea que, por medio de la construcción de un espacio de diálogo, se traten temas de relevancia para la persona privada de libertad, y que con esto se humanice la permanencia en los 
URL: http://www.revistas.una.ac.cr/index.php/dialogo/index

CORREO ELECTRÓNICO: universidadendialogo@una.cr

DOI: https://doi.org/10.15359/udre.9-1.6

centros penitenciarios y su vinculación con el sistema penitenciario de Costa Rica como institución jurídica y social. Vera Grabe (2008) señala:

La paz como paradigma nos permite construir paz, reconociendo y desarticulando la violencia como una lógica de exclusión que desciframos en los esquemas mentales y culturales basados en la linealidad, la negación, la dualidad, la separación, la polarización, la discriminación, los prejuicios y estereotipos, para optar por lógicas y maneras de pensar y conocer desde la no-exclusión: la complejidad, la horizontalidad, la integralidad, la pluralidad, la paradoja. Nos permite reconocer que la sociedad es ambigua, mezcla de pazy de violencia, para fortalecer la primera y desarmar la otra. Y nos ayuda a superar lógicas de retaliación de víctima-victimario, de amigo enemigo, de fronteras y límites entre seres humanos, que sólo reproducen los circuitos perversos de violencia (p. 16).

Nuestro trabajo va encaminado a lograr un diálogo entre privados de libertad, pretende aportar consecuentemente a las estrategias nacionales, con el objetivo de validar una política cada vez más humanista, democratizada e inclusiva, sin perder de vista las condiciones reales, en este caso, de la población privada de libertad. El resultado de las experiencias académicas, de investigación y extensión universitaria desde el proyecto va a generar el conocimiento y la experiencia para buscar unir esfuerzos para implementar una estrategia que fortalezca las capacidades de convivencia pacífica y cultura de paz en la población privada de libertad.

\section{Expresiones artísticas para la construcción de una cultura de paz}

Nuestro planteamiento en su enfoque teórico se fundamenta en que el movimiento es inherente al ser humano y está inmerso en nuestro entorno, por lo que consideramos que incorporar en el proceso actividades lúdicas, basadas en el movimiento y la expresión, permitirá dar un mayor significado a situaciones específicas que facilitarán el conocimiento interno y su entorno e ir construyendo su propia realidad.

$\mathrm{Al}$ respecto y siguiendo en la misma línea teórica que fundamenta la propuesta, Stokoe (2008) señala lo siguiente:

Consideró inseparable el concepto de "expresión corporal-danza" y partió del principio de que todo movimiento puede ser una danza y poseer una carga expresiva. La entendió como una conducta espontánea inherente al ser humano, como un lenguaje mediante el cual la persona 
expresa sensaciones, emociones, sentimientos y pensamientos, con el cuerpo, integrándola de esta manera a sus otros lenguajes expresivos, como el habla, el dibujo, la pintura, la escritura, la poesía, etc. (p. 1)

Igualmente, Fux (2004) define que el movimiento y la vivencia con nuestro cuerpo y con los demás producen cambios que se reflejan positivamente en nuestra salud y en la forma de relacionarnos, pensar, sentir y actuar. Debido a ello, la persona permite que su cuerpo tome el control de sus emociones y exprese lo que está sintiendo en ese momento, ayudando así a enriquecer la experiencia y el desarrollo personal a través de las prácticas artísticas. Además, hace alusión a que, a través de la expresión artística y el movimiento creativo, se desarrollan habilidades para socializar, fortalece la autoestima, permite el control de las emociones y estimula los espacios de integración colectiva, ya que busca la reflexión de lo cotidiano por medio del lenguaje de movimiento.

Con base en este contexto teórico, el arte del movimiento será la principal herramienta artística a utilizar, con el fin de abordar la socialización del individuo bajo el enfoque propio de cultura de paz. Las actividades serán dirigidas de forma creativa, sin códigos establecidos, con el fin de expresar sensaciones, sentimientos, pensamientos, e inclusive narrar historias propias o vivencias grupales. Las prácticas nos conducirán gradualmente a una transformación interior y social que poco a poco permitirá variar hábitos, relaciones, disciplina, diversión, organización, entre otros.

La propuesta busca que las personas privadas de libertad posean un espacio de construcción, donde se manifieste la expresión artística y se tengan beneficios como conocerse mejor, desarrollar una actitud más sensible y expresar ideas por medio del movimiento, ya que muchas veces la expresión verbal puede verse distorsionada y el diálogo de movimiento facilita la comunicación. Como acciones propias de una estrategia que conlleve a ese marco integral de búsqueda de soluciones o alternativas de mejora desde lo social y cultural, la aplicación de un enfoque pedagógico y práctico de técnicas artísticas se convierte en un elemento fundamental y potenciador en la implementación de la estrategia para el fomento de una cultura de paz.

\section{Conclusiones finales}

En forma muy concreta, las acciones emprendidas dentro de la estrategia con el fin de alcanzar los objetivos planteados en el proyecto consistieron en la realización de actividades tales como talleres, charlas, juegos y presentaciones 
URL: http://www.revistas.una.ac.cr/index.php/dialogo/index

CORREO ELECTRÓNICO: universidadendialogo@una.cr

DOI: https://doi.org/10.15359/udre.9-1.6

artísticas, bajo un enfoque ludopedagógico en cinco áreas que, de acuerdo con nuestro planteamiento, deberían caracterizar el enfoque de una cultura de paz, tales como: historia de vida, mecanismos de negociación, comunicación libre y transformadora, resolución alternativa de conflictos, buen vivir y cultura de paz. Así, por ejemplo, mediante las diversas y variadas actividades que desarrollamos se estimularon rasgos y cualidades que conforman la identidad de las personas privadas de libertad: el sentido de pertenencia, el desarrollo de su propia afectividad y equilibrio emocional, para contribuir a una autoestima positiva y a la aceptación de sí mismo.

Paralelamente a la realización de estos talleres y como parte de la estrategia, se creó una coreografía en la que participó un grupo seleccionado de privados de libertad con el fin de recrear situaciones y experiencias propias del ser humano a través de la expresión artística y del lenguaje de movimiento, de tal manera que el participante pudiera exteriorizar sus propios sentimientos, emociones y vivencias. Para la ejecución de la coreografía se llevaron a cabo sesiones de trabajo para la sensibilización del tema y la búsqueda de los movimientos corporales que mejor lo reflejaran, posteriormente, con la práctica de la coreografía el temor a expresarse fue disminuyendo. Fux y Bensignor (2004), en su libro Qué es la danzaterapia, señalan al respecto:

Cuando bailamos, expresamos no solo la belleza, sino también los miedos, la rabia, la angustia, el dolor. Cada uno de esos estados son personajes que viven dentro de nosotros y que pugnan por salir con la misma intensidad con que nos resistimos, muchas veces, a dejarlos aflorar o, tal vez, reconocerlos como propios. Y es a través de la danza, más que de la palabra, como logran encontrar esa salida. (p. 14)

Un aprendizaje enriquecedor a partir de la ejecución de la coreografía fue el conversatorio grupal sostenido con todos los participantes, en el que cada uno de forma espontánea expresó como en algún momento de su vida experimentaron situaciones similares que les pudieron haber afectado en su proceso de socialización.

Logramos que esta población participante tuviera una muy buena dinámica, en donde el diálogo al final de cada una de las sesiones nos ha permitido percibir el impacto de los estados emocionales vividos por cada uno de los participantes y revelados por ellos mediante sus expresiones, generando a la vez la toma de conciencia del carácter compartido de los sentimientos, los cuales les producen una gran satisfacción e identificación. 
Desde el punto de vista metodológico, el planteamiento propone como medio idóneo para llevar a cabo los componentes de expresión artística y cultura de paz un enfoque cualitativo con el método de investigación-acción-participativa (IAP), ya que cuando la expresión oral se vuelve insuficiente, el movimiento y la expresión artística se tornan en un canal de expresión, en el que es posible manifestar la interioridad.

La metodología se fundamenta en tres momentos inspirados en la educación popular: la práctica promovida por juegos y actividades, la teorización de la realidad (construir conocimiento desde la reflexión individual y en grupo, superando lo anecdótico) y la práctica transformando la realidad (aplicar el conocimiento adquirido sobre la realidad para operar sobre ella, cambiando actitudes y acciones).

Finalmente, se puede mencionar que la experiencia obtenida hasta la fecha evidencia mejoras en el entorno y la vida de la población participante en las actividades organizadas. Por ello, valoramos la implementación de la estrategia mediante la organización de actividades de expresión artística, que dan paso a la creatividad de la persona privada de libertad, que le permite expresarse libremente y enriquecerse de forma espiritual, mental, emocional y social a partir de la experiencia vivida, partiendo de sus características naturales y de un proceso lúdico.

\section{Referencias bibliográficas}

Comisión Nacional de Rectores (2017). Programa Estado de la Nación: II Informe Estado de la Justicia (2 ${ }^{\text {da }}$ ed.). San José, Costa Rica: Servicios Gráficos AC.

Dell' Anno, A. y Teubal, R. (2006). Resignificando lo grupal en el trabajo social. Buenos Aires, Argentina: Espacio.

Fux, M. y Bensignor, B. (2004). Qué es la danzaterapia: preguntas que tienen respuestas. Buenos Aires: Lumen.

Geertz, C. (1989). El impacto del concepto de cultura en el concepto de hombre. En C., Geertz, La interpretación de las culturas. Barcelona: Gedisa.

Grabe, V. (2008). Paciculturas - Paz como pedagogía para la vida. Revista Virtual Perspectivas, $\mathrm{N}^{\circ}$ 13. Recuperado de www.revistalanumero13. con/publicación-n1/pacicultura.html 
Naciones Unidas (1999). Declaración y Programa de Acción sobre una Cultura de Paz. [En línea] (A/RES/53/243), 6 de octubre de 1999

Stokoe, P. (2008). La expresión corporal-danza. Recuperado de educadoresyarte. blogspot.com/2008/08/la-expresion-coorporal-danza-segun.html

Vargas, S. (2016). La construcción social de la violencia. Inventio, 12(27), $27-$ 33. Recuperado de http://inventio.uaem.mx/index.php/inventio/article/ view/163/407

\section{Referencias consultadas}

Coppens, F., Van De Velde, H.(2005). Técnicas de Educación Popular. Nicaragua, Estelí. CURN/CICAP

Díaz, R., Quiros, K. (2017). Hacinamiento penitenciario costarricense: Definición y control constitucional. Revista jurídica IUS Doctrina, número uno, 10(1), 1-28. Recuperado de https://revistas.ucr.ac.cr/ index.php/iusdoctrina/article/view/23006/29512

Gabarrón, L., Hernández L. (1994). Cuadernos Metodológicos: Investigación participativa. Madrid. Centro de Investigaciones Sociológicas.

Muñoz, F., Molina B. (2009). Una Cultura de Paz compleja y conflictiva. La búsqueda de equilibrios dinámicos. Recuperado en http://www.ugr. es/ revpaz/articulos/Una_Cultura_de_Paz_compleja_y_conflictiva.html 